\title{
Detection and imaging of moving targets in wide band SAS using fast time backprojection combined with space time processing
}

\author{
M.I. Pettersson \\ Department of Signalprocessing, School of Engineering \\ Blekinge Institute of Technology \\ Soft Center, Ronneby, Sweden \\ Phone: +46 457385728 Email:mtp@bth.se \\ V. Zetterberg \\ Department of Signalprocessing, School of Engineering \\ Blekinge Institute of Technology \\ Soft Center, Ronneby, Sweden \\ Phone: +46 704411220 Email: viktoria.zetterberg@amlab.se \\ I. Claesson \\ Department of Signalprocessing, School of Engineering \\ Blekinge Institute of Technology \\ Soft Center, Ronneby, Sweden \\ Phone: +46 457385717 Email: ingvar.claesson@bth.se
}

\begin{abstract}
This paper present a method to combine SAS (Synthetic Aperture Sonar) imaging of stationary targets with moving target detection and imaging. The proposed method uses a likelihood ratio for moving target detection in a wide band (WB) SAS system. For this paper, WB is defined as any systems having a large fractional bandwidth, i.e. an ultra wide frequency band combined with a wide antenna beam. The developed method combines time domain fast backprojection SAS processing methods with moving target detection using space-time processing.

In the paper defocusing and detection of moving targets are investigated. Both the trajectory and the location of the moving target is given by mathematical expressions.
\end{abstract}

\section{INTRODUCTION}

In the latest years there has been a growing interest in time domain fast backprojection techniques for image formation in Synthetic Aperture Sonar (SAS) [1] and in synthetic aperture radar (SAR). The main reason for the interest is that these methods are claimed to handle the motion compensation more easily than the FFT based methods. Another reason for the growing interest is that the fast backprojection methods now are claimed to be as fast as the FFT methods $[2,3]$.

The domain of different Fast Backprojection Methods can be categorized based upon the number of stages used in the processing. In global backprojection [4] there is only one stage, the range data are directly used to form an image, and no intermediate beamforming stage is used. In twostage algorithms there is an intermediate step with beamforming over sub-apertures, an example of a two stage algorithm is Fast Backprojection Algorithm [5]. In multi-stage algorithms beams are formed of beams formed at the stage before. Examples of multi stage algorithms are for Quadtree Backprojection [2] and Fast Factorized Backprojection [3]. For beamforming in sonar the algorithm [6] has similar structure.

When a SAR or SAS image are formed the moving target will be unfocused and displaced. Using an array antenna with different spatial input channels will give the possibility to discriminate a moving target from clutter. This has for many years been an important topic in the radar community. In the latest years there have been a growing interest to combine moving target detection with SAR processing for low frequency SAR systems [12]. At low frequencies a large integration angle is needed to detect and image the moving target. Therefore new algorithms have been developed for moving target detection [7] and imaging. Because backprojection has been found to be efficient for WB systems the proposed algorithm given in this paper combines backprojection with space time processing. Due to similarities between SAS and low frequency SAR we propose a algorithm originally developed for low frequency SAR to detect moving targets SAS systems.

\section{FOCUSING}

In this this work we consider a moving platform with the transmitter and receiver array, and a moving target which we want to detect and image. As normal in this type of study we assume a linear motion of the platform and the targets.

A non-moving target on the seabed will at the sensor cause a hyperbolic range migration as the platform passes by. This hyperbolic migration is well known from SAR and SAS literature. If there is a moving target on the seabed it will also cause a hyperbolic range migration at 
the platform. However this hyperbolic function will have another shape then the hyperbolic function of the stationary target. The unmatched distance will cause unfocusing and dislocation of the moving targets in the SAS image, as seen in $[8,9]$. To focus the moving target the shape of it's hyperbola has to be considered. It has been shown that all moving targets will be focused considering the proper relative speed [10].

We will in this paper consider moving targets in SAS data. Therefore we will not only restrict the consideration of moving targets on the seabed but rather targets moving in the water masses. The range migration of a moving target with the freedom to move in all coordinate directions is given by

$R(t)=\sqrt{\left(v_{p l} t-\left(x_{0}+v_{x} t\right)\right)^{2}+\left(y_{0}+v_{y} t\right)^{2}+\left(z_{0}+v_{z} t\right)^{2}}$

where $x_{0}, y_{0}$ and $z_{0}$ is the position of the moving target at $t=0, v_{x}, v_{y} v_{z}$ is the speed of the moving target in the three directions and $v_{p}$, is the speed of the platform moving on the $\mathrm{x}$-axis. This distance can be rewritten to

$R(t)=\sqrt{\gamma_{m}^{2}\left(v_{p l} t-X_{0}\right)^{2}+Y_{0}^{2}}=\sqrt{\gamma_{m}^{2}\left(x_{p l}-X_{0}\right)^{2}+Y_{0}^{2}}$

by identification of (2.1) in (2.2) we get

$\gamma_{t}=\sqrt{\frac{\left(v_{p l}-v_{x}\right)^{2}+v_{y}^{2}+v_{z}^{2}}{v_{p}^{2}}}$

$X_{0}=\frac{x_{0}\left(v_{p l}-v_{x}\right)-y_{0} v_{y}-z_{0} v_{z}}{\gamma_{t}^{2} v_{p l}}$

$Y_{0}=\sqrt{x_{0}^{2}+y_{0}^{2}+z_{0}^{2}-\frac{\left(x_{0}\left(v_{x}-v_{p l}\right)+y_{0} v_{y}+z_{0} v_{z}\right)^{2}}{\gamma_{t}^{2} v_{p l}^{2}}}$

which means that to focus any moving target we have to consider the relative speed between the platform and the moving target. The position in the focused SAS image will be given by the image coordinates $X_{0}$ and $Y_{0}$. Notice that this also holds for non-moving targets where $\gamma_{t}=1$, $X_{0}=x_{0}$, and $Y_{0}=\sqrt{x_{0}^{2}+y_{0}^{2}+z_{0}^{2}}$.

When a moving target is present in the SAS image and we process the image considering another relative speed, the moving target will appear as unfocused. We now determine the curvature of the moving target, having a relative speed coefficient $\gamma_{t}$ but is imaged in a SAS process assuming $\gamma_{p}$. In this case all targets moving in all directions will be focused if they have a relative speed given by $\gamma_{p}$. From the curvature the image impulse response can be found.

The SAS process will place energy of the moving target in the SAS image considering distance and Doppler. At time $t$ the position of the moving target will be given by

$$
\begin{aligned}
& r_{t}(t)=r_{p}(t) \\
& r_{t}^{\prime}(t)=r_{p}^{\prime}(t)
\end{aligned}
$$

where $r_{t}$ is the range of the moving target and $r_{p}$ is the assumed range used in the image formation

$$
\begin{aligned}
& r_{t}(t)=\sqrt{\gamma_{t}^{2}\left(v_{p l} t-X_{t}\right)^{2}+Y_{t}^{2}} \\
& r_{p}(t)=\sqrt{\gamma_{p}^{2}\left(v_{p l} t-X_{p}\right)^{2}+Y_{p}^{2}}
\end{aligned}
$$

Solving this equation (2.6-2.7) at time $t$ will give us the appearance at time $t$ in the SAS image. The trajectory $\left(X_{p}, Y_{p}\right)$ of the target will then be given by considering all $t$ used in the azimuth integration. In image coordinates the trajectory is given by

$$
\frac{Y_{p}^{2}}{Y_{t}^{2}}+\frac{\gamma_{p}^{2} \gamma_{t}^{2}}{\gamma_{t}^{2}-\gamma_{p}^{2}} \frac{\left(X_{t}-X_{p}\right)^{2}}{Y_{t}^{2}}=1
$$

If $\gamma_{p}<\gamma_{t}$ the equation is an equation describing an elips with axis $Y_{t}$ and $\sqrt{\gamma_{t}^{2}-\gamma_{p}^{2}} / \gamma_{p} \gamma_{t}$ and centre point at $\left(X_{t}, 0\right)$. If $\gamma_{p}=\gamma_{t}$ the image is focused so $X_{p}=X_{t}$ and $Y_{p}=Y_{t}$.

If $\gamma_{p}>\gamma_{t}$ the equation is a hyperbola with transverse axis $2 Y_{p}$ and conjugate axis $2 \sqrt{\gamma_{t}^{2}-\gamma_{p}^{2}} / \gamma_{p} \gamma_{t}$. The shapes are described and illustrated in Fig. 1. 


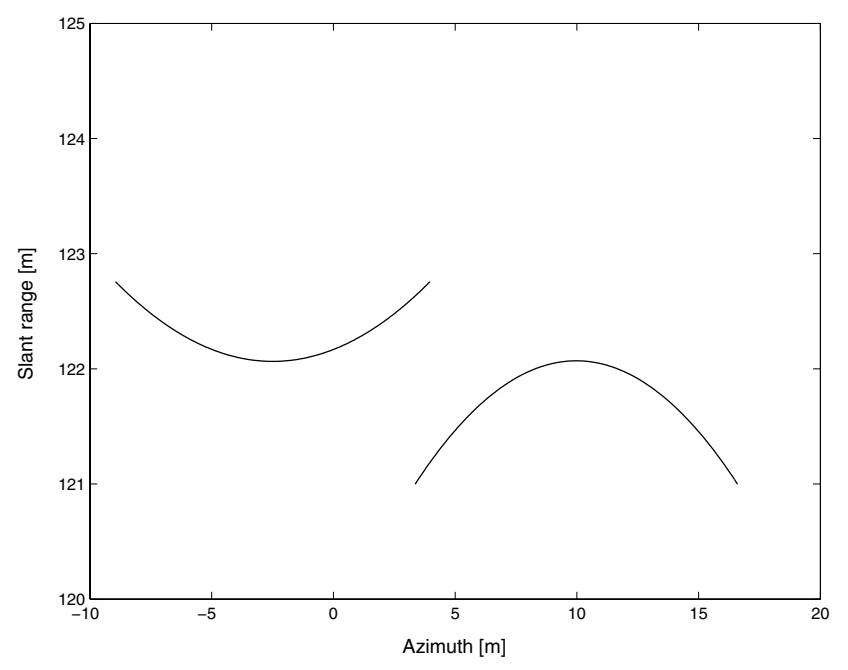

Fig. 1. Two moving targets processed at non correct relative speed. In the left of the figure there is a moving target with speed $(1,0.5,1) \mathrm{m} / \mathrm{s}$ and on the right a moving target with speed $(-1,0.5,1) \mathrm{m} / \mathrm{s}$. The platform speed is $(10,0,0) \mathrm{m} / \mathrm{s}$ and the integration angle is $30^{\circ}$. Relative speed coefficient of the two moving targets are approximately 0.9 and 1.1. The two targets perform hyperbolic and eliptical trajectory due to the incorrect relative speed.

\section{IMAGE FORMATION}

In the two stage backprojection algorithms the total synthetic aperture is divided in equally sized sub-apertures. In each sub-aperture there are many beams formed for all different directions over the image area. In next stage these beams from different sub-apertures are used to form the image.

In the first stage in multistage algorithms beams are formed over a very small aperture. These beams are used in next stage to form new beams with higher resolution. At every stage new beams are formed using the beams in the stage before. This procedure can be described as an iterative process. In the last stage the computed beams are used to form an image. In this paper we are interested to show the possibility to detect or imaging moving targets. Let $y\left(r, \gamma_{p}, m\right)$ be a beam formed in a stage in the fast backprojection processing [7]. The image formation is given by

$h\left(X, Y, \gamma_{p}\right)=\sum_{m=1}^{M} y\left(r\left(X, Y, \gamma_{p}\right), \gamma_{p}, m\right)$.

If the relative sub-aperture length (which is a combination of real aperture and the relative speed) is not to big we can use the approximation

$$
y\left(r\left(X, Y, \gamma_{p}\right), \gamma_{p}, m\right) \approx y\left(r\left(X, Y, \gamma_{p}\right), 1, m\right)
$$

In this case we can use beams calculated for one speed rather at the exact relative speed. At some stage in the multi-stage algorithms this approximation is not valid any more, and the next stages in the algorithm has to consider a more accurate relative speed. As the resolution or integration time increases a more accurate relative speed is needed. In [9] a two stage backprojection algorithm is used to focus moving targets. In the paper, beams at first stage are processed at ground speed. In the second stage the ground speed beams are combined considering relative speed to final resolution.

\section{FAST BACKPROJECTION IN AN ANTENNA ARRAY}

To detect a moving target an antenna array often is used. By using space time adaptive processing the spatial correlated noise from the stationary targets can be suppressed while all the non-stationary such as moving target will be detected. In this article we propose an along track array with a transmitting antenna separated from the receiving antenna array. However because there will be bistatic measurements between the transmitting antenna and the receiving antennas we define an effective antenna phase centre. The effective antenna centre approximates the bistatic wave from two displaced antennas as a monostatic wave from one antenna located in the middle of the transmitting and receiving antenna. How this approximation affects SAR processing and moving target detection is given in $[11,12]$. The antenna configuration has a total of $L$ channels and the separation between the first effective antenna center to the effective antenna centre of channel $l$ is $d_{l}$. Fast backprojection methods can easily handle the bistatic wave originated in the array antenna, so in the processing there is no need to compensate the bistatic wave according to $[11,12]$. However in the space time processing we have to consider the distance, and in this work we assume the elements in the array to be equally separated given by $d$. The sub-aperture for channel $l$ is given by

$h_{l}\left(X, Y, \gamma_{p}\right)=\sum_{m=1}^{M} y_{l}\left(r\left(X, Y, \gamma_{p}\right), \gamma_{p}, m\right)$

\section{MOVING TARGET DETECTION}

When a beam is processed with a fast backprojection method it has a direction connected to Doppler speed of the non-moving targets. When a moving target is present the appearance of that target will be shifted due to the separation of true speed and processing speed. The moving target will be processed to the same beam as non-moving targets at another position, as given in section II. We will in this section use $\varphi^{\prime}$ for the true target position and $\varphi$ for the direction where the target appears in the processing.

If there is no moving target present then the sub-aperture beam only contain clutter and noise while if a moving target is present, the sub-aperture beams consist of a moving target, clutter and noise. The clutter noise has spatial dependence while noise has no spatial dependency. 
The clutter and noise are assumed to be independent of each other.

To detect a moving target we want to use as big aperture as possible to increase the signal clutter noise ratio (SCNR). At the same time we know that the synthetic aperture used in the clutter suppression can not be as big as the total integration time do to the strong clutter and the non stationarity of the clutter [7]. The optimum is very dependent on system parameters. However the multi-stage algorithms are very tolerant in this case and we can choose to do the clutter suppression at any stage in the SAS processing.

Assume that we have selected the stage were the clutter suppression should be done. We also assume that this stage is before the image formation stage. From the available beams $y_{l}\left(r, \gamma_{p}, m\right)$ we calculate the beam in wave domain $\tilde{y}_{l}\left(k_{n}, \varphi_{m}\right)$. Where $k_{n}$ is the radius in wave domain and $\varphi_{m}$ is the direction angle. Using all $L$ channels in the array we can form a long vector $\tilde{\mathbf{Y}}$ consisting of all beams connected to a moving target. To detect a target we have two hypotheses in the test, $\mathbf{H}_{1}$ with a target present and

$\mathbf{H}_{\mathbf{0}}$ with no target present. The vector $\tilde{\mathbf{Y}}$ will then for the two hypotheses be written as

$\tilde{\mathbf{Y}}=\left[\begin{array}{c}\tilde{y}_{1}\left(k_{0}, \varphi_{1}\right) \\ \tilde{y}_{2}\left(k_{0}, \varphi_{1}\right) \\ \vdots \\ \tilde{y}_{L}\left(k_{0}, \varphi_{1}\right) \\ \tilde{y}_{1}\left(k_{1}, \varphi_{1}\right) \\ \vdots \\ \tilde{y}_{L}\left(k_{N-1}, \varphi_{1}\right) \\ \vdots \\ \tilde{y}_{L}\left(k_{N-1}, \varphi_{M}\right)\end{array}\right]=\left\{\begin{array}{cc}\tilde{\mathbf{q}}+\tilde{\mathbf{n}} & \text { under } \mathbf{H}_{0} \\ \tilde{\tilde{\mathbf{A}}}+\tilde{\mathbf{q}}+\tilde{\mathbf{n}} & \text { under } \mathbf{H}_{1}\end{array}\right.$

Where $\tilde{\tilde{\mathbf{A}}}$ is the vector connected to the moving target, $\mathbf{q}$ is the vector connected to the correlated noise and $\mathbf{n}$ is the uncorrelated noise. To test if a target is present we use the likelihood ratio test given by

$\Lambda=\frac{P\left(\tilde{\mathbf{Y}} \mid \mathbf{H}_{1}\right)}{P\left(\tilde{\mathbf{Y}} \mid \mathbf{H}_{\mathbf{0}}\right)}$
Assuming both the correlated and the non correlated noise to be Gaussian gives us the probability density functions under $\mathbf{H}_{\mathbf{0}}$ with no target present

$P\left(\tilde{\mathbf{Y}} \mid \mathbf{H}_{\mathbf{0}}\right)=\frac{1}{(2 \pi)^{N M}|\tilde{\mathbf{C}}|} e^{-\tilde{\mathbf{Y}}^{H} \tilde{\mathbf{C}}^{-1} \tilde{\mathbf{Y}}}$

and under $\mathbf{H}_{1}$ with a target present

$P\left(\tilde{\mathbf{Y}} \mid \mathbf{H}_{\mathbf{1}}\right)=\frac{1}{(2 \pi)^{N M}|\tilde{\mathbf{C}}|} e^{-(\tilde{\mathbf{Y}}-\tilde{\tilde{\mathbf{A}}})^{H} \tilde{\mathbf{C}}^{-1}(\tilde{\mathbf{Y}}-\tilde{\tilde{\mathbf{A}}})}$

where $\tilde{\mathbf{C}}$ is the covariance matrix of $\tilde{\mathbf{Y}}$. We now assume a well calibrated system and that the scattering is caused by a point target having the same scattering strength $S_{0}$ at all frequencies and directions, but with a random scattering phase $\phi_{0}$

$\tilde{\tilde{\mathbf{A}}}=s_{0} e^{j \phi_{0}} \tilde{\mathbf{A}}$

where $\tilde{\mathbf{A}}$ is the steering vector given by

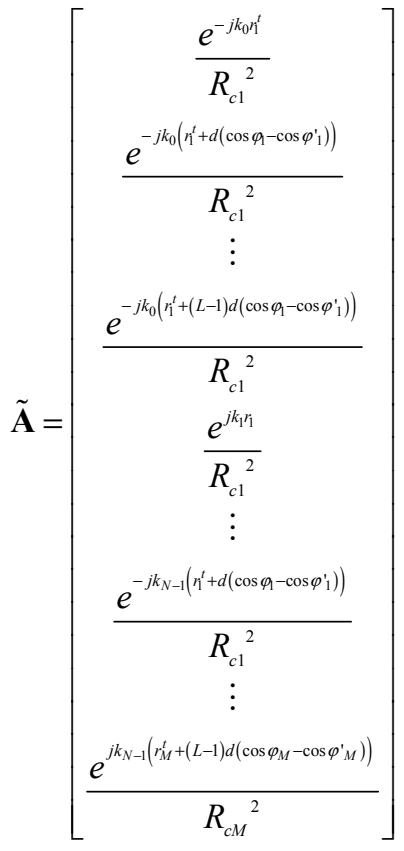

Where $r_{m}^{t}$ is the location of the target in the beam, $\varphi_{m}$ is the beam where the target is located, $\varphi_{m}^{\prime}$ the true direction to the target, and $d$ is the distance between the antenna arrays assuming a linear antenna. Where $r_{m}^{t}$ and $\varphi_{m}$ are 
connected to the range migration part given in section II and dependent on $X_{0}, Y_{0}, \gamma_{m}$ and $\gamma_{p}$.

Since is $\phi_{0}$ is random and distributed uniformly between 0 and $2 \pi$ the test variable [13] is given by

$$
\left|\tilde{\mathbf{A}}^{H} \tilde{\mathbf{C}}^{-1} \tilde{\mathbf{Y}}\right|^{2}\left\{\begin{array}{l}
>\lambda \text { decision for } \mathbf{H}_{1} \\
<\lambda \text { decision for } \mathbf{H}_{\mathbf{0}}
\end{array}\right.
$$

Assuming that the resolution cell in the beam $y\left(r, \gamma_{p}, m\right)$ contains many scatters, and by that, that the covariance matrix can be simplified to a large matrix with zeros and on the diagonal a sub-matrix given by

$$
\overline{\mathbf{C}}_{n m}=E\left[\left(\overline{\mathbf{q}}_{n m}+\overline{\mathbf{n}}_{n m}\right)\left(\overline{\mathbf{q}}_{n m}+\overline{\mathbf{n}}_{n m}\right)^{H}\right]
$$

where $\overline{\mathbf{q}}_{n m}$ and $\overline{\mathbf{n}}_{n m}$ are the clutter and noise matrix for the antenna array given one wavenumber in direction $m$ and frequency $n$. Using this simplified covariance we get test looking similar to the image formation in (3.1)

$$
\begin{aligned}
& \left|\tilde{\mathbf{A}}^{H} \tilde{\mathbf{C}}^{-1} \tilde{\mathbf{Y}}\right|^{2}=\left|\sum_{m=1}^{M} \frac{1}{R_{c m}^{2}} \sum_{n=0}^{N-1} e^{j k r_{m}^{\prime} \mathbf{\mathbf { A }}_{m m}^{H}} \overline{\overline{\mathbf{A}}}_{m}^{H}\left(\varphi_{m}^{\prime}\right) \overline{\mathbf{C}}_{m m}^{-1} \overline{\mathbf{Y}}_{m m}\right|^{2}= \\
& =\mid \sum_{m=1}^{M} \frac{1}{R_{c m}^{2}} y_{c}\left(r_{m}^{t}, m,\left.\varphi_{m}^{\prime}\right|^{2}=\left\{\begin{array}{l}
>\lambda \text { decision for } \mathbf{H}_{1} \\
<\lambda \text { decision for } \mathbf{H}_{0}
\end{array}\right.\right.
\end{aligned}
$$

\section{DISCUSSION}

We have in this paper showed a method that we claim can be used in SAS data to detect moving targets. The test image to test if a moving target is present at $\left(X_{t}, Y_{t}\right)$ with heading $\alpha$ and relative speed coefficient $\gamma_{t}$ is given by

$$
\begin{aligned}
\left|h\left(X_{t}, Y_{t}, \gamma_{t}, v\right)\right|^{2}=\left|\sum_{m=1}^{M} \frac{1}{R_{c m}^{2}} y_{c}\left(r_{m}^{t}, m, \varphi_{m}^{\prime}\right)\right|^{2}= \\
=\left\{\begin{array}{l}
>\lambda \text { decision for } \mathbf{H}_{1} \\
<\lambda \text { decision for } \mathbf{H}_{0}
\end{array}\right.
\end{aligned}
$$

In this test there are some parameters that have to be selected to get the best performance in the SAS-STAP processing. The first question is at which stage in the fast backprojektion should clutter suppression be applied to merge all array element beams to one beam? How long in total can the integration time of the target be and how big are the steps in between tested $\gamma_{p}$.
The question, at which stage in the fast backprojection the STAP process should be applied is probably only dependent on the platform motion, the stationarity of the clutter and the mathematical approximation made in the SAR processor. The reason for this is that the clutter signal in many cases is so much stronger than the target signal. The clutter suppression also has to be performed in an early stage, due to non linear behaviour of the clutter. In [7] it was found that the optimum clutter suppression appeared approximately when the synthetic sub-aperture is in the size of the far-field condition. However, we believe that this is very system related issue.

After the clutter suppression is performed the clutter suppressed beams can be used for sub-aperture beams with better resolution or be used for image formation. Probably the clutter suppression has to be done in an early state so the resolution is increased through some algorithm stages before the image stage is reached. If a moving target is detected with the wrong relative speed the proper relative speed can be estimated from the shape of the moving target target using (2.8).

In reality some moving targets do not move with the same speed and direction during the time the target is illuminated by the SAS system. The linear motion approximation is limited and the limitation is given by the target motion and the system performance. However these targets can be detected or imaged with lower resolution using part of the illumination time for image construction. Linear motion can always be applied over shorter periods of time and when using backprojection methods, it is very easy to adjust the integration time needed.

\section{REFERENCES}

[1] S.M. Banks and H.D. Griffiths, "The use of fast factorised backprojection for synthetic aperture sonar", Proceedings of the Sixth European Conference on Underwater Acoustics, ECUA '2002, Gdansk, Poland, pp529-534.

[2] J. McCorkle and M. Rofheart, "An order $N^{2} \log (\mathrm{N})$ backprojector algorithm for focusing wide-angle widebandwidth arbitary-motion synthetic aperture radar", Proc. of SPIE Conference on Radar Sensor Technology, Vol. 2747, Orlando, 1996, pp. 25-36

[3] L.M.H. Ulander, H. Hellsten and G. Stenström, "Synthetic-Aperture Radar Processing using Fast Factorised Back-Projection", IEEE Transactions on Aerospace and Electronic Systems, Vol. 39, No. 3, July 2003, pp.760-776

[4] L.E. Andersson, "On Determination of a Function from Spherical Averages", SIAM J. of Appl. Math., Vol. 19, No. 1, 1988, pp 214-341

[5] A.F. Yegulalp, "Fast backprojection Algorithm for Synthetic Aperture Radar", Proc. of 1999 IEEE Radar Conference, Waltham, Massachusetts, 1999, pp. 60-65 
[6] K.M. Houston, "A fast beamforming algorithm", IEEE oceans Conference, Brest France, Vol. 1, pp 211-216, 1994

[7] M.I. Pettersson, "Detection of moving targets in wideband SAR", IEEE Trans. Aerospace and Electronic systems, vol 40, no.3, pp. 780-796, Juli 2004

[8] R. K. Raney: "Synthetic Aperture Imaging Radar and Moving Targets", IEEE Trans. Aerospace and Electronic Systems, Vol. AES-7, No. 3, 1971, pp. 499505

[9] M.I. Pettersson, "Focusing of Moving Targets in an Ultra-wide band SAR GMTI System", Proc. of EUSAR 2000, 3rd Europian Conference on Synthetic Aperture Radar, Germany, 2000, pp. 837-840

[10]H. Hellsten and L.M.H. Ulander, "Airborne Array Aperture UWB UHF-Motivation and System Consideration", Proceedings of the 1999 IEEE Radar Conference -Radar to the next millenium, Waltham, 1999, pp. 47-53

[11]M. Soumekh, "Moving Target Detection in Foliage Using Along Track Monopulse Synthetic Aperture Radar Imaging", IEEE Transactions on Image Processing, vol. 6, pp. 1148-1162, 1997

[12]M.I. Pettersson: "Extraction of moving ground targets by a bistatic ultra-wideband and -widebeam SAR system", IEE Proc. Radar, Sonar and Navigation, Vol. 148, No. 1, 2001, pp. 35-40

[13]W.S. Chen and I.S. Reed, "A New CFAR Detection Test for Radar", Digital Signal Processing, vol. 1, no. 4, pp. 198-214, 1991 